\title{
Grado de satisfacción en la prestación de servicio de restauración en los puertos de la provincia de El Oro
}

\section{Degree of satisfaction in the restoration services in the ports of El Oro Province}

Enrique Germán Pérez Maldonado ${ }^{1}$ Rosa Ángela Cedillo Ordoñez ${ }^{2}$ \& Melissa Paulina Calle Iñiguez ${ }^{3}$.

Resumen: La actividad turística y la gastronomía van intrínsecamente ligados, porque cuando turistas visitan un nuevo destino requieren de servicio de restauración para satisfacer sus necesidades, es de ahí que nace la gran interrogante ¿Cuál es el lugar ideal para degustar delicias gastronómicas típicas de la provincia de El Oro? En este contexto se ha realizado una investigación con el objetivo de analizar el grado de satisfacción del consumidor/cliente en la prestación de servicios de alimentos en los puertos de la provincia de El Oro. Se estableció seis criterios de evaluación: servicio, producto, instalación/mobiliaria, imagen, seguridad y precio. La metodología fue una encuesta en escala de Likert, aplicada a las poblaciones de Machala, Santa Rosa y Huaquillas. No solo es necesario ofertar deliciosos platillos, sino, también conocer al consumidor y sus deseos a través del uso de instrumentos de satisfacción que Puerto Bolívar, Puerto Jeli y Puerto Hualtaco no utilizan; sin embargo, los resultados demuestran un bajo nivel de insatisfacción, pero es necesario que haya innovación en los restaurantes.

Palabras clave: Puerto, servicio, restauración, satisfacción, cliente.

\begin{abstract}
Tourist activity and gastronomy are inoculate, because when tourists visit new destination requires of services restauration for conclude needs. This produce a question, which is the best place for a taste of typical gastronomy of EI Oro? This research has the objetive of analyze the degree of satisfaction of the customer for the purveyance of food service in the ports of El Oro. It was apply six assessment criteria: service, product, facilities, image, security and prices. The methodological, was a survey made in likert scale, this was apply to population of: Machala, Santa Rosa and Huaquillas. It do not only necessary to have delicious dishes, but then know the customers and their wishes through satisfaction instruments, that Puerto Bolivar, Puerto Jeli and Puerto Hualtaco do not use. The results show declive percentage of insatisfaction, but it is necessary that there innovation in the restaurants.
\end{abstract}

Key Words: Port, service, restoration, satisfaction, customer.

(Presentado: 14 de agosto de 2019. Aceptado: 4 de noviembre de 2019)

\footnotetext{
1 Estudiante en Administración de Hotelería y Turismo, Universidad Técnica de Machala, Ecuador. egperezm_estQutmachala.edu.ec

${ }^{2}$ Estudiante en Administración de Hotelería y Turismo, Universidad Técnica de Machala, Ecuador. racedillo_estQutmachala.edu.ec

${ }^{3}$ Doctora en Ciencias Administrativas, Magister en Planificación Turística y Docente Lcda. en Administración de Empresas Turísticas y Hoteleras. Universidad Técnica de Machala, Ecuador. mpcallequtmachala.edu.ec
} 


\section{INTRODUCCIÓN}

Dentro de la división geográfica del Ecuador se sitúa la provincia de El Oro, siendo una de sus fortalezas la variedad de pisos ecológicos, que proceden desde playas, ríos y bosques. Está conformada por 14 cantones, teniendo como ciudad capital a Machala. El sustento económico principal de la provincia se debe a su producción primaria: agrícola y aurífera; asimismo acuícola, teniendo tres puertos, como son: Puerto Bolívar, ubicado en el cantón Machala, caracterizado por ser uno de los principales puertos del país donde su mayor exportación es el banano y cacao, a partir de esta actividad productiva se generó la prestación de servicios y alimentos; asimismo, se encuentra Puerto Jeli perteneciente al cantón Santa Rosa, conocido por su riqueza gastronómica y cultivos de camarón, los cuales poseen la mayor exportación a nivel nacional, y por último se sitúa Puerto Hualtaco que se lo encuentra en el cantón Huaquillas en zona fronteriza con el hermano país del Perú. Lugares propicios para el consumo de platillos a base de mariscos debido a su gran variedad y factible obtención, además que promueven la economía dentro del país y permiten un consecuente desarrollo de sus cantones.

La diversa oferta gastronómica de los tres puertos de la provincia de El Oro genera turismo interno, a pesar de ello, hay una carencia de data sobre los deseos, gustos y preferencias del consumidor, entre otros; que son necesarios para corroborar la opinión que tiene el turista interno acerca de la calidad y servicio del producto que consume, es así que Olviera (2010) hace mención que para procurar mantener y atraer turistas todo depende de la satisfacción que éste reciba y que su efecto puede repercutir a otras personas. Por lo tanto, es importante hacer uso de herramientas que midan las experiencias del consumidor para poder establecer metas, pero ninguno de los puertos lo aplica.

El saber la opinión del consumidor permite establecer fortalezas y debilidades del sector, debido a que ayudan a establecer una mejora continua. Una cualidad de los puertos de la provincia de El Oro en función a los productos alimenticios (frutos del mar) es que son obtenidos por pescadores de forma artesanal. Esta característica permite a los restaurantes de los puertos obtener un producto fresco. No obstante, se ha podido determinar que existe un desconocimiento sobre el grado de satisfacción en la prestación de servicio de restauración. Por ello, se plantea en la presente investigación determinar el grado de satisfacción en la presentación del servicio, producto, instalación e inmobiliaria, seguridad, imagen y precio en los restaurantes de los Puertos de la Provincia de El Oro.

Se puede corroborar que en los restaurantes de los tres puertos de la provincia de El Oro según los datos obtenidos por la escala de likert demuestra un bajo porcentaje de insatisfacción, a pesar de ello hay falencias de innovación, con respecto al servicio, producto, seguridad e imagen, asimismo se debería abarcar nuevos nichos de mercado, para solventarse en días no tan productivos. Se podría establecer una mejora si los dueños de los restaurantes se enfocarán más en sus comensales, Aguilar, Yépez, Esteban \& Sierra (1999) hacen mención que no es conveniente buscar soluciones donde no se ha implicado un esfuerzo por saber que satisface a los clientes y como es catalogado frente a la competencia.

\section{METODOLOGÍA}

Se aplicó el método cuantitativo y deductivo, el cual permitió medir el grado de satisfacción que tiene la población que consumen servicios gastronómicos, en los restaurantes de los tres puertos de la provincia de El Oro:

Población de estudio

Para la realización de las encuestas se tomó de referencia la población de la parroquia Machala, parroquia Santa Rosa y parroquia Huaquillas, donde según el Instituto Nacional de Estadísticas y Censos (2010), la detalla a continuación:

\section{Tabla No 1. Población de estudio.}

\begin{tabular}{ll}
\hline Parroquia & Población \\
Machala & 241.606 \\
Santa Rosa & 52.863 \\
Huaquillas & 48.285 \\
Total & 342.754
\end{tabular}

Fuente: Elaborado por autores. INEC.

\section{Muestra}

Se determinó una muestra poblacional empleando la Fórmula Infinita, para lo cual se planteó como resultado 380 personas con un nivel de confianza del $95 \%$ y un error esperado del $5 \%$. 


$$
n=\frac{Z^{2} * p * q}{e^{2}}
$$

Z: Nivel de confianza.

$\mathrm{p}$ : Porcentaje de la población que tiene el atributo deseado. q: Porcentaje de la población que no tiene el atributo deseado $=1-p$

e: Error de estimación máximo aceptado.

$\mathrm{n}$ : Tamaño de la muestra.

$$
n=\frac{\left(1,95^{2}\right)(0,5)(0,5)}{\left(0,05^{2}\right)} n=\frac{(3,8025 * 0.25)}{0,0025} n=\frac{0,950625}{0,0025} n=380,25
$$

\section{Instrumento metodológico}

Se empleó la encuesta, la cual tomó como referencia de medición la Escala de Likert, debido a que esta herramienta permite conocer el grado de satisfacción de las personas mediante la valorización perceptiva del encuestado. Acorde al porcentaje obtenido de los tres puertos de la provincia de El Oro se estableció un valor para cada parámetro:

$\begin{array}{ll}\text { 1) } & \text { Sobresaliente } \\ \text { 2) } & \text { Muy bueno } \\ \text { 3) } & \text { Bueno } \\ \text { 4) } & \text { Regular } \\ \text { 5) } & \text { Malo }\end{array}$

Se consideraron seis criterios de valoración para determinar el grado de satisfacción, los cuales se detallan a continuación:

$\begin{array}{ll}\text { 1) } & \text { Servicio } \\ \text { 2) } & \text { Producto } \\ \text { 3) } & \text { Instalación y mobiliaria } \\ \text { 4) } & \text { Seguridad } \\ \text { 5) } & \text { Imagen } \\ \text { 6) } & \text { Precio }\end{array}$

\section{ANÁLISIS Y DISCUSIÓN DE RESULTADOS}

Se entiende por satisfacción al estado de euforia que muestra una persona al concluir de manera positiva la terminación de un deseo, la misma que puede variar en intensidad por diferentes factores. Es así que Besanilla, Rodríguez \& Ponce (2012), mencionan aspectos, como: culturales, personales, sociales y psicológicos, los mismos que pueden condicionar el comportamiento del consumidor.

A continuación, se muestran los resultados obtenidos referente al criterio de valoración de servicio:

Figura $N^{\circ}$ 1. Criterio Servicio: Atención al cliente.

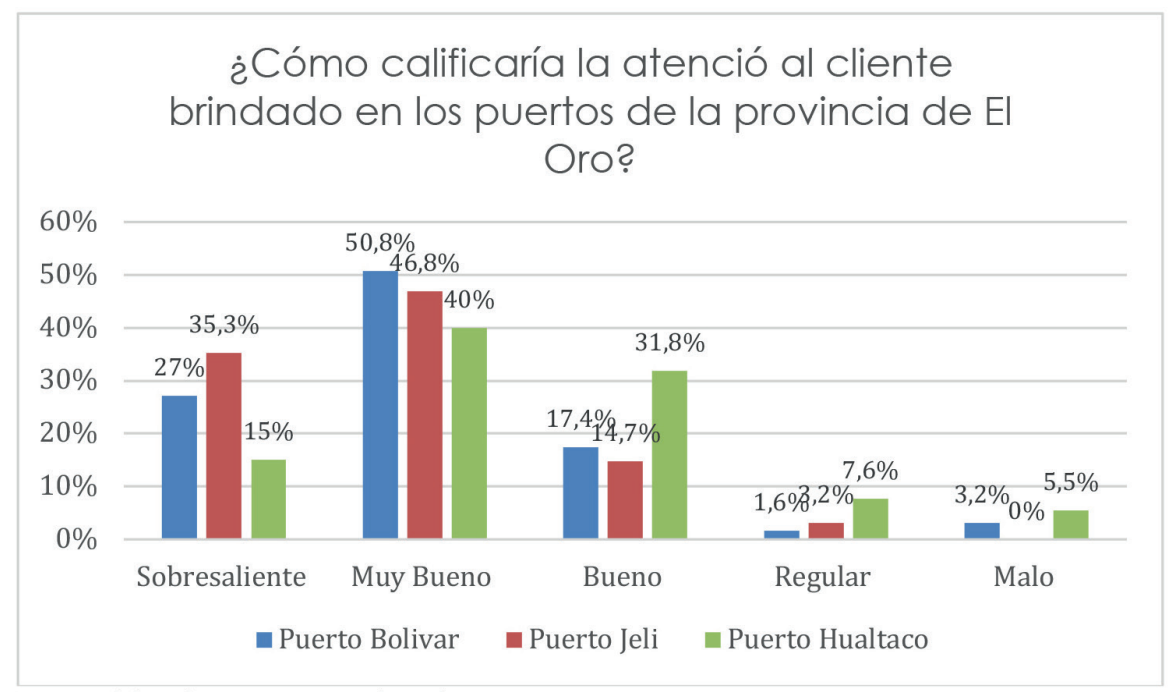

Fuente: Elaborado por autores, Trabajo de campo 2019. 
"El servicio y la atención de calidad son el reflejo del compromiso de quienes integran una institución orientada al cliente, usuario o público en general” (Estrada, 2007). Esto debido que es el consumidor quien genera el capital suficiente para el funcionamiento de un emprendimiento, el cual si este aplica un buen trato puede incidir en el regreso de su clientela. Acorde a los resultados, un $27 \%$ considera que la prestación de servicios en Puerto Bolívar es sobresaliente, a diferencia de Puerto Jeli que obtuvo un 35,3\% y Puerto Hualtaco el 15\%; en cambio en muy bueno tienen el $50,8 \%, 46,8 \%$ y $40 \%$ : muestran un total como bueno del $17,4 \%, 14,7 \%$ y el $31,8 \%$; en regular poseen el $1,6 \%, 3,2 \%$ y $7,6 \%$; en el último parámetro el primer y último puerto indican un $3,2 \%$ y $5,5 \%$ como malo.

Figura $N^{\circ} 2$. Criterio Servicio: Tiempo de espera.

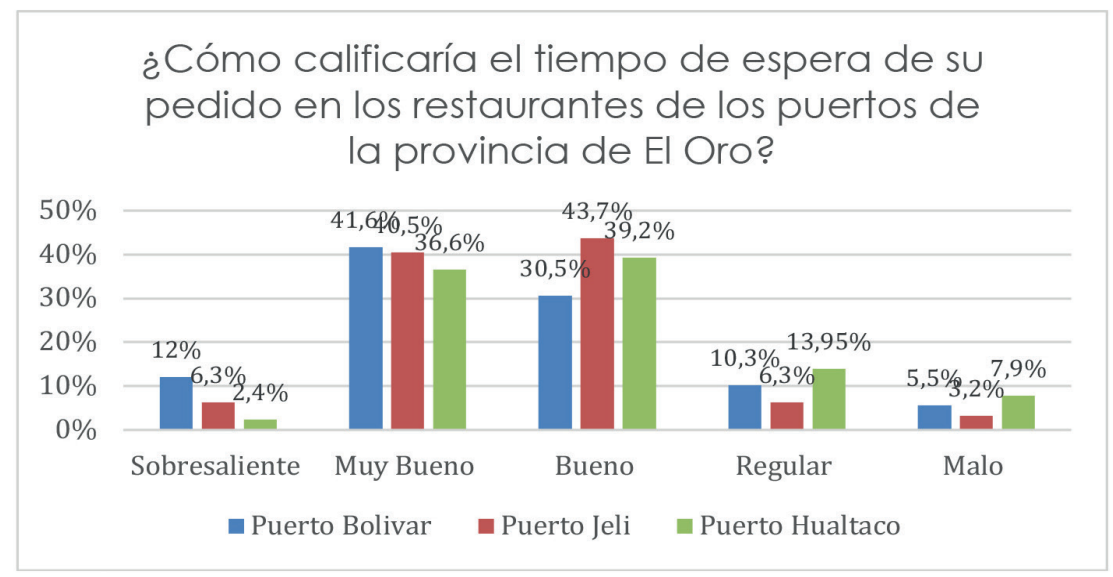

Fuente: Elaborado por autores. Trabajo de campo 2019.

"El bien denominado turismo es muy complejo, pues incluye los bienes y los servicios que consume el turista durante el periodo de tiempo que está fuera de su hogar" (Narváez \& Fernández, 2010). El viajero siempre busca lo más conveniente con respecto a lo que desea, sin que intervengan con las obligaciones o responsabilidades que debe cumplir, y a la hora de esperar un pedido entre me- nos demore mayor es su satisfacción. En este caso Puerto Bolívar posee un $12 \%$ como sobresaliente, Puerto Jeli un $6,3 \%$ y Puerto Hualtaco presenta el $2,4 \%$; en muy bueno tienen porcentajes similares del $41,6 \%, 40,5 \%$ y $36,6 \%$; lo mismo sucede en bueno que poseen el $30,5 \%$, $43,7 \%$ y $39,2 \%$; obteniendo en regular el $10,3 \%, 6,3 \%$ y $13,95 \%$, y en el parámetro malo poseen el 5,5\%, 3,2\% y 7,9\%.

Figura $N^{\circ}$ 3. Criterio Servicio: Proceso de pago y facturación.

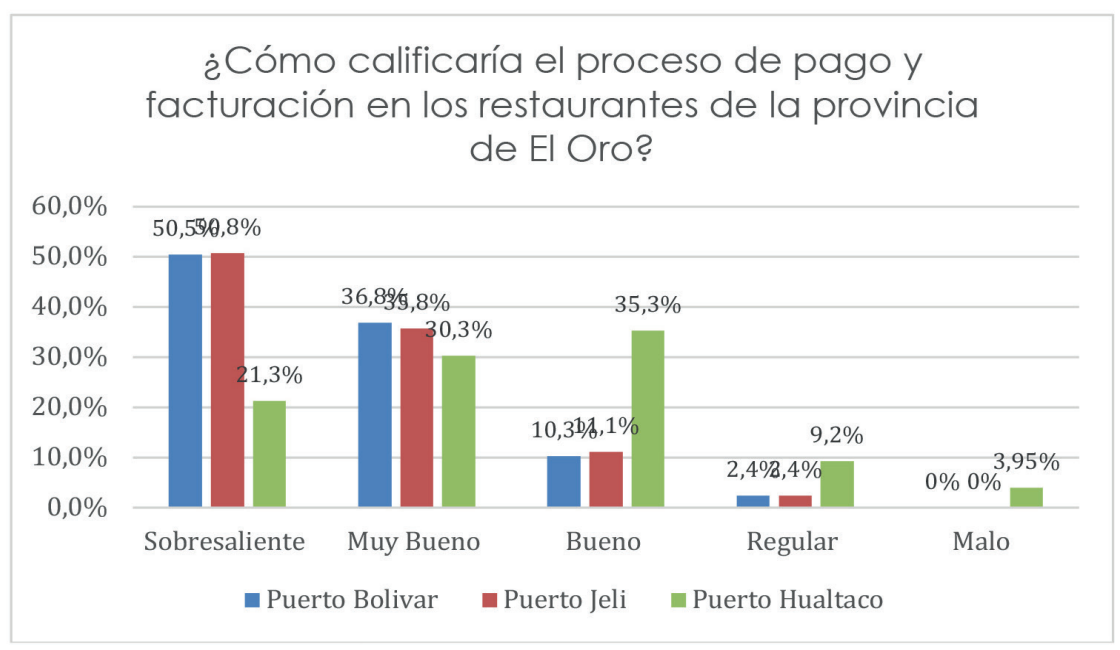

Fuente: Elaborado por autores. Trabajo de campo 2019. 
Maldonado (2015), indica que a pesar de los diversos medios de pago existentes en el mercado en Latinoamérica el $91 \%$ de las transacciones son hechas en efectivo, pero debido a la continua desaparición del cheque como forma de cancelación, la tarjeta de crédito ha incrementado su preferencia. Es por ello que es necesario que los restaurantes tengan por lo menos dos facilidades de pago, para que el turista tenga más comodidades al cancelar su orden, Puerto Bolívar obtuvo como resultado en sobresaliente el $50,5 \%$, Puerto Jeli el
$50,8 \%$ y Puerto Hualtaco un 21,3; mostrando poca diferencia en muy bueno con el $36,8 \%$, $35,8 \%$ y $30,3 \%$; en cambio en bueno varía del $10,3 \%, 11,1 \%$ y $35,3 \%$; asimismo como en el anterior parámetro los dos primeros puertos presentan los porcentajes más bajos en regular del 2,4\% y el tercer puerto del 9,2\%; además que es el único que tiene un $3,95 \%$ en malo.

Asimismo, el criterio de valorización Producto da como resultado lo siguiente:

Figura $N^{\circ}$ 4. Criterio Producto: Frescura de materia prima.

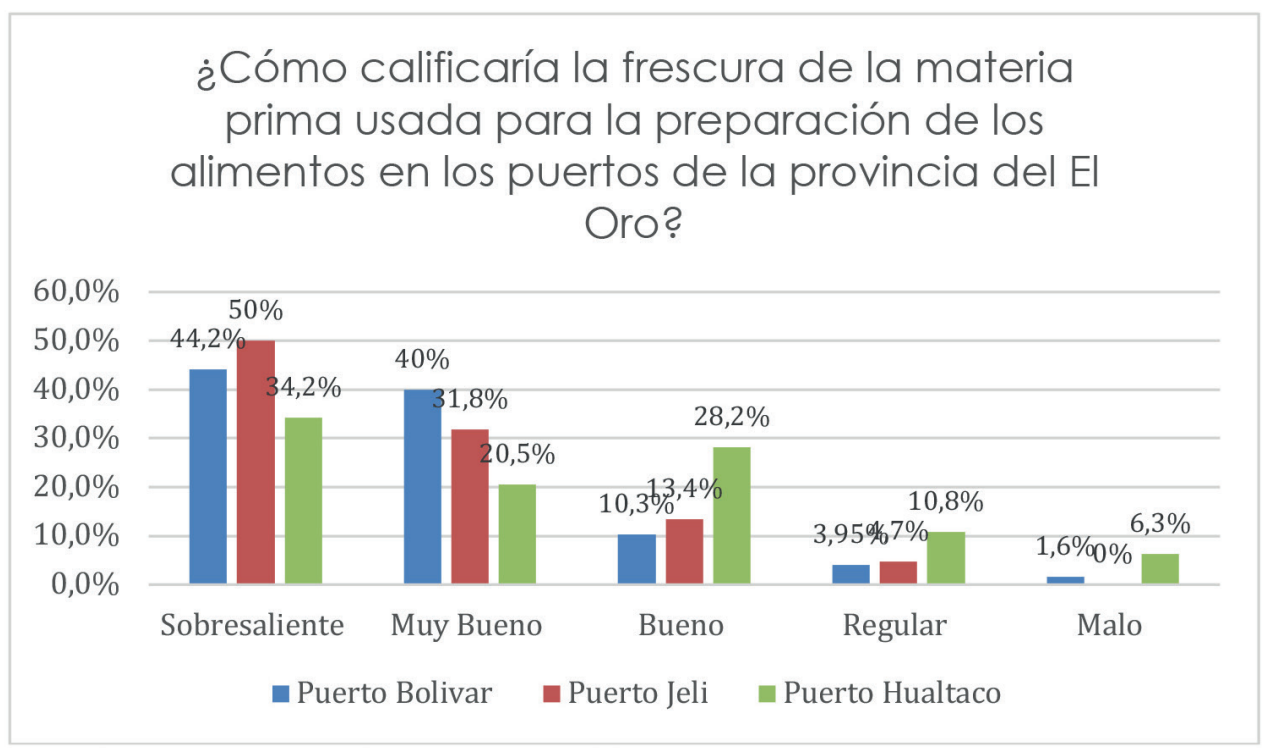

Fuente: Elaborado por autores. Trabajo de campo 2019.

En lo que respecta a mariscos se debe tener mucho cuidado con lo que se ingiere, sobre todo si la materia prima no ha sido bien manipulada o no es fresca, Roberts y Stadler (2009) mencionan que desde la obtención del espécimen puede contaminarse con bacterias, virus y parásitos que pueden producir enfermedades de alto riesgo. Puerto Bolívar obtuvo como resultado en sobresaliente el $44,2 \%$, Puerto Jeli el $50 \%$ y Puerto Hualtaco el $34,2 \%$; en cambio en muy bueno varía desde $40 \%$, 31,8\% y $20,5 \%$; en el parámetro bueno se observa un $10,3 \%, 13,4 \%$ y $28,2 \%$; prosiguiendo en regular con el $3,95 \%, 4,7 \%$ y $10,8 \%$; por último, el $1,6 \%$ y $6,3 \%$ del primer y tercer puerto consideran que es Malo. 
Figura $N^{\circ}$ 5. Criterio Producto: Sabor, textura y aroma.

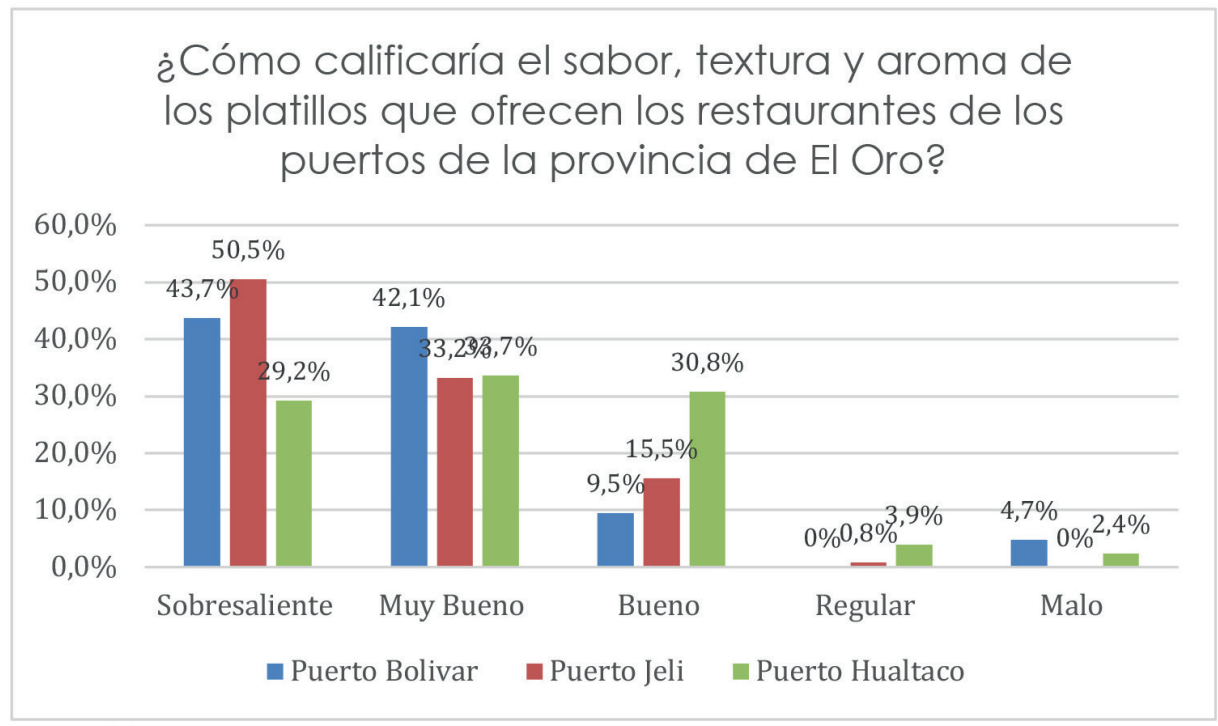

Fuente: Elaborado por autores. Trabajo de campo 2019.

Delgado (2001) menciona que al hacer uso de nuestros sentidos al comer, se puede encontrar particularidades pertenecientes a un grupo humano, esto debido que la gastronomía está ligada a nuestra historia social. Por ende, las formas de preparación y cocción de los alimentos pueden variar, incluso en sitios de comida localizados en una misma zona, con respecto al sabor, textura y aroma de los platillos de los restaurantes en los puertos de la provincia de El Oro, Bolívar obtuvo un 43,7\% como sobresaliente,
Jeli logró un $50,5 \%$ y Hualtaco el $29,2 \%$; en cambio en muy bueno presentan un $42,1 \%, 33,2 \%$ y $33,7 \%$; habiendo porcentajes en bueno del 9,5\%, 15,5\% y $30,8 \%$; sin embargo en regular muestran un $0 \%, 0,8 \%$ y $3,9 \%$; por último un calificativo del $4,7 \%$ y $2,4 \%$ como ,alo de Puerto Bolívar y Puerto Hualtaco.

Con respecto a la Instalación y mobiliaria se obtuvo lo siguiente:

Figura № 6. Criterio Instalación y mobiliaria: Aseo.

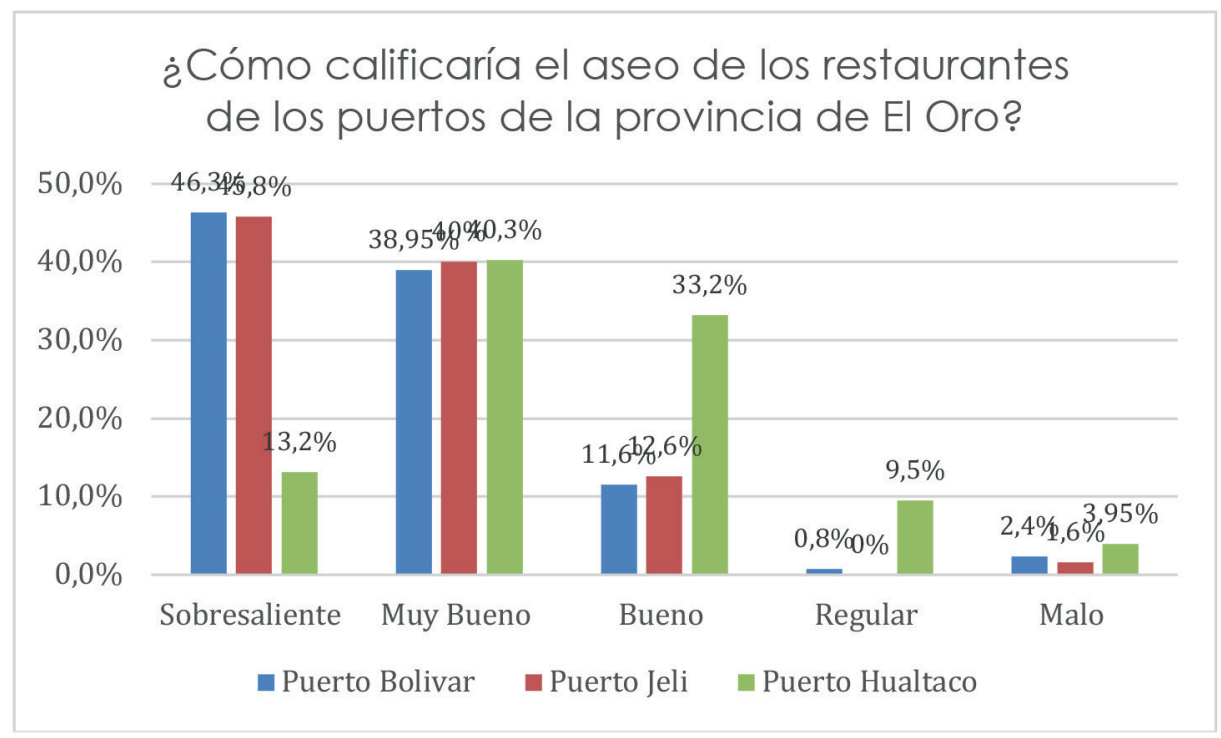

Fuente: Elaborado por autores. Trabajo de campo 2019. 
Rubio (2014) afirma que, para lograr mantener las instalaciones, equipos o utensilios en condiciones óptimas para su consecuente uso, se debe llevar a cabo un correcto procedimiento de limpieza y desinfección, debido que estas dos acciones permiten eliminar la suciedad visible y reducir los microorganismos. Los signos de aseo en la restauración son muestra de alimentos propicios para su consumo y acorde a los resultados
Puerto Bolívar obtuvo un 46,3\% como sobresaliente, Puerto Jeli un $45,8 \%$ y Puerto Hualtaco un porcentaje del $13,2 \%$; pero en el parámetro muy bueno existe una similitud entre los tres del $38,95 \%, 40 \%$ y $40,3 \%$; en cambio en bueno presentan el $11,6 \%, 12,6 \%$ y $33,2 \%$; habiendo como regular el $0,8 \%, 0 \%$ y $9,5 \%$ y con una cantidad mínima del 2,4\%, 1,6\% y 3,95\% que piensan que el aseo de los puertos es malo.

Figura $N^{\circ} 7$. Criterio Instalación y mobiliaria: Comodidad.

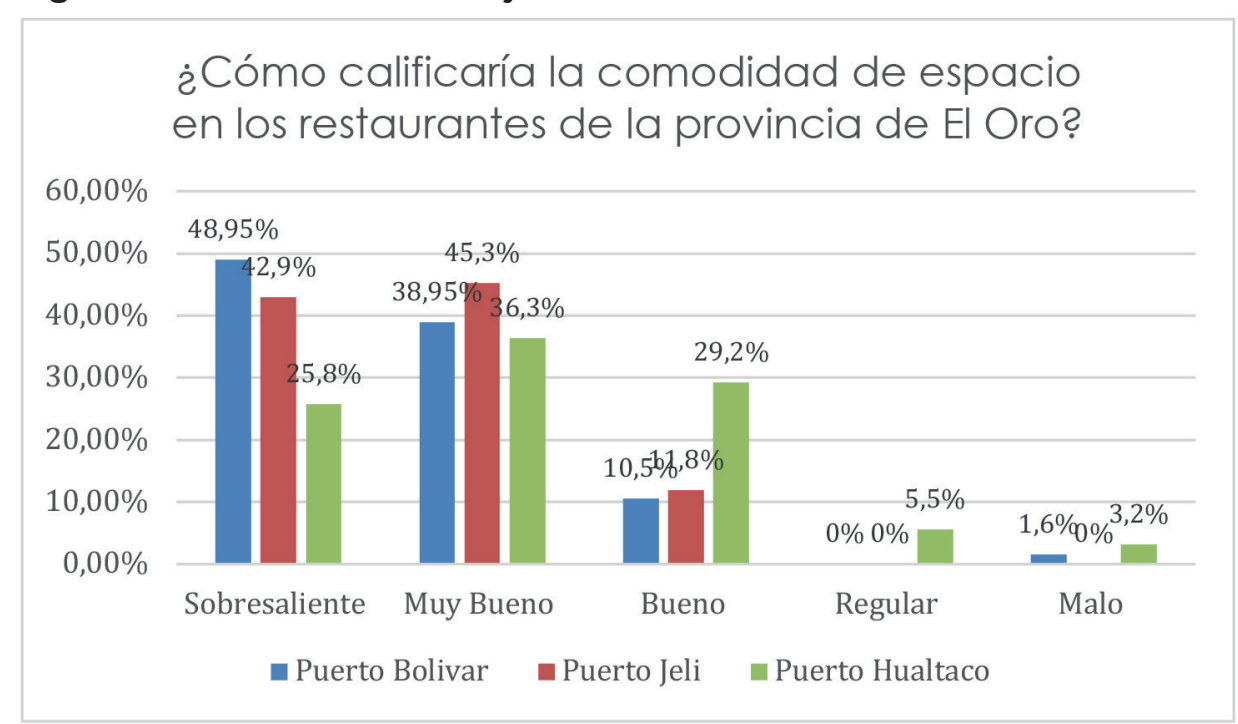

Fuente: Elaborado por autores. Trabajo de campo 2019.

Un restaurante se compone, principalmente, del área de cocina y servicio, siendo este último el espacio de interacción directa con el comensal, el cual si no es optimizado puede incurrir a obstruir las actividades del mesero o en caso de proximidad de mesas provocar que el cliente pierde privacidad. Los tres puertos por lo general presentan este problema debido a que la demanda supera la capacidad de oferta en función a las instalaciones los días feriados, sin embargo, acorde a los resultados Puerto Bolívar obtuvo como sobresaliente el $48,95 \%$, Puerto Jeli el $42,9 \%$ y Puerto Hualtaco el $25,8 \%$; como muy bueno tienen porcentajes del $38,95 \%, 45,3 \%$ y $36,3 \%$; en cambio en bueno el $10,5 \%$, $11,8 \%$ y $29,2 \%$; pero en regular los dos primeros puertos poseen un $0 \%$ y el último un 5,5\%; en el parámetro malo Bolívar tiene el 1,6\% y Hualtaco el $3,2 \%$.

En el criterio Seguridad se consiguió lo siguiente: 
Figura $N^{\circ}$ 8. Criterio Seguridad: Restaurantes.

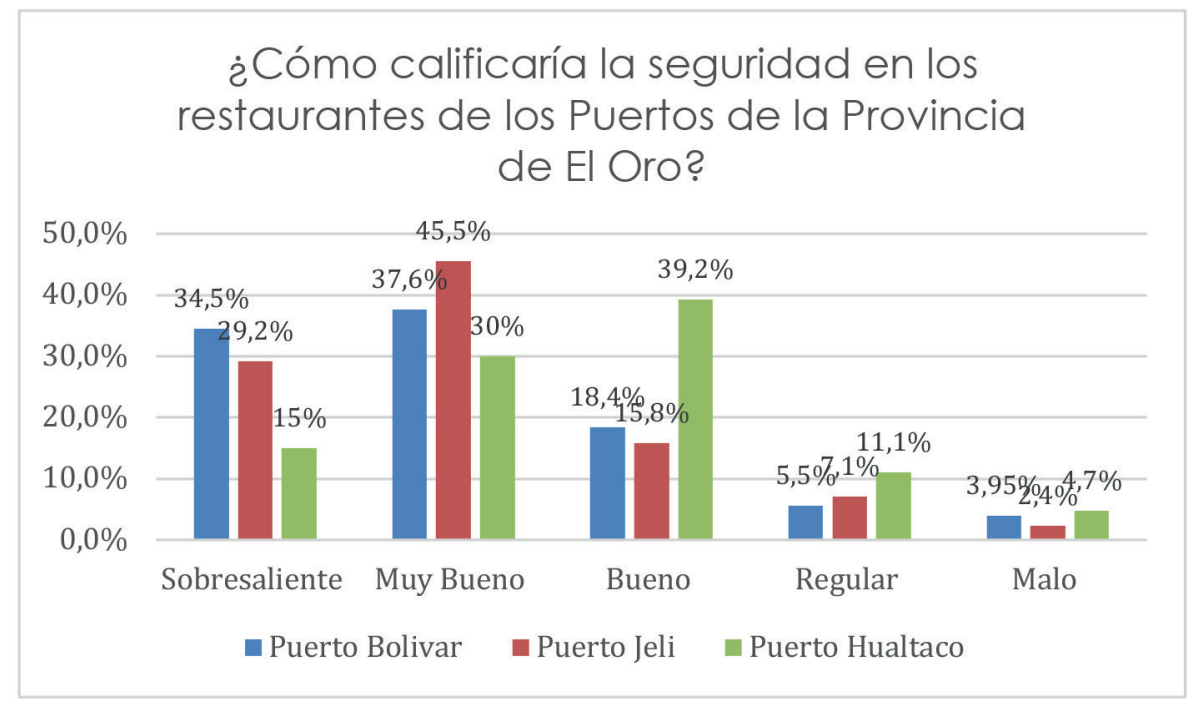

Fuente: Elaborado por autores. Trabajo de campo 2019.

Según Crespo (2019) los sitios con aumento de índices delictivos en la provincia de El Oro, son el cantón fronterizo Huaquillas y la parroquia Puerto Bolívar, en Machala. Por ello es necesario que los establecimientos implementen medidas de seguridad como: cámaras de vigilancia, alarmas, guardianía o mantener un control en la entrada del restaurante. Es así que mediante los resultados se puede corroborar que la seguridad dentro de los establecimientos de Puerto Bolívar el 34,5\% lo considera sobresaliente a diferencia de Jeli que tiene el $29,2 \%$ y Hualtaco el $15 \%$; como muy bueno tienen el $37,6 \%, 45,5 \%$ y $30 \%$; en cambio en bueno el $18,4 \%, 15,8 \%$ y $39,2 \%$; habiendo en regular porcentajes del $5,5 \%, 7,1 \%$ y $11,1 \%$ y en malo el $3,9 \%$, $2,4 \%$ y $4,7 \%$.

Figura $N^{\circ}$ 9. Criterio Seguridad: Estacionamientos.

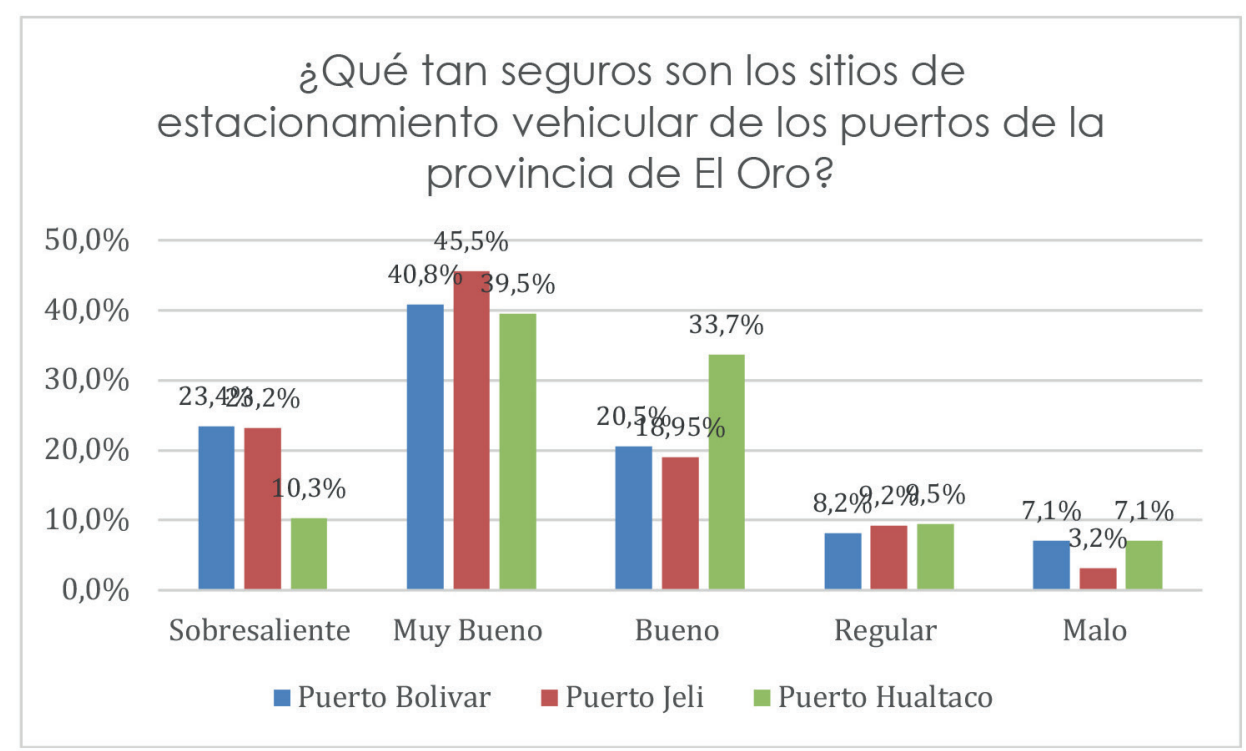

Fuente: Elaborado por autores. Trabajo de campo 2019. 
"El estacionamiento es una condición evidentemente indispensable en todo sistema de transporte vial” (Bull, 2003), sobre todo si el conductor ha llegado a su destino y ya no requiere movilizarse, el inconveniente que radican estos espacios en los puertos de la provincia de El Oro es su informalidad, por ende, existe más riesgo de ser víctima de un antisocial. Acorde a los resultados el $23,4 \%$ consideran como sobresaliente la seguridad en los parqueaderos de
Puerto Bolívar, un 23,2\% Puerto Jeli y 10,3\% Puerto Hualtaco; en cambio como muy bueno tienen el $40,8 \%, 45,5 \%$ y $39,5 \%$; el calificativo de bueno es del $20,5 \% 18,95 \%$ y $33,7 \%$; en regular con porcentajes similares del 8,2\%, 9,2\% y $9,5 \%$; por último, en malo poseen un $7,1 \%, 3,2 \%$ y $7,1 \%$.

El criterio de valorización Imagen tiene como resultado lo siguiente:

\section{Figura $N^{\circ} 10$. Criterio Imagen: Corporativa.}

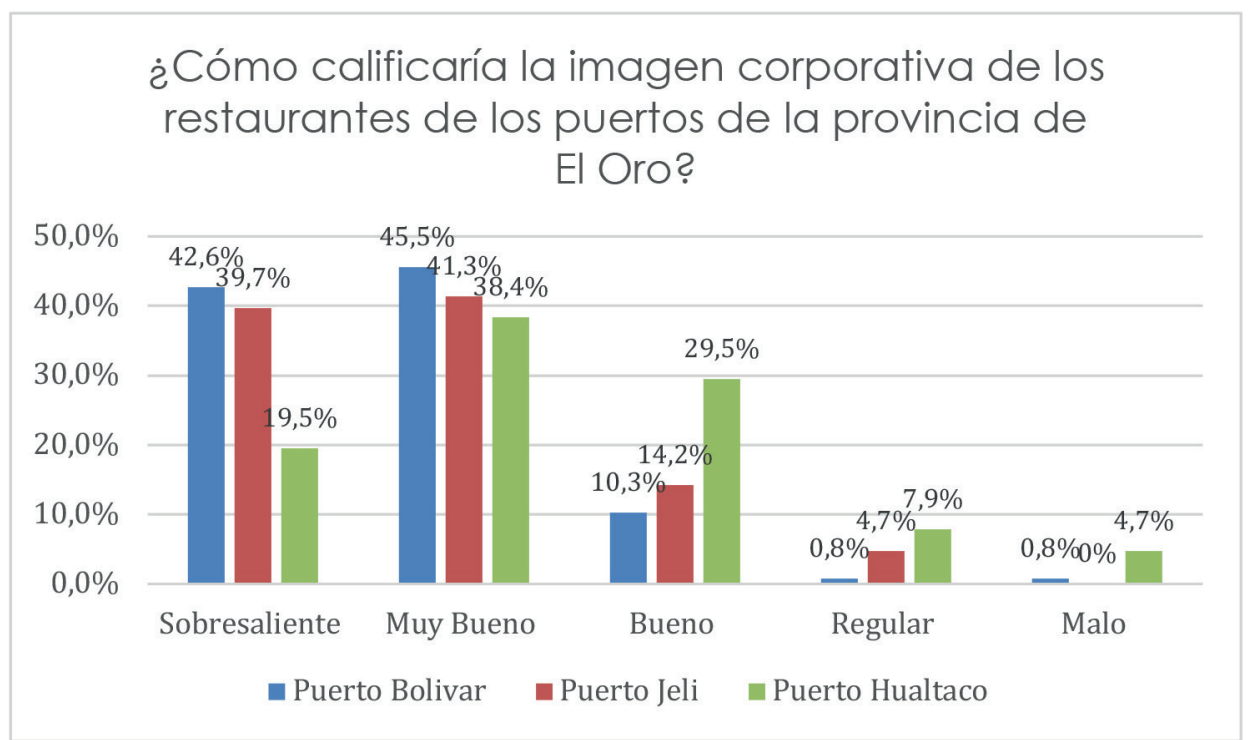

Fuente: Elaborado por autores. Trabajo de campo 2019.

La imagen corporativa es la carta de presentación de una determinada entidad, esto permite al consumidor dar una idea de todo el entorno, además que "se ha convertido en la piedra angular de todo el proceso comunicacional de empresas e instituciones" (Galán , 2008), por ende, si proyectas algo positivo quien lo recepte replica esa impresión, según los resultados
Puerto Bolívar obtuvo en sobresaliente el $42,6 \%$, Puerto Jeli un $39,7 \%$ y Puerto Hualtaco el $19,5 \%$; como muy bueno tuvieron el $45,5 \%, 41,3 \%$ y $38,4 \%$; en cambio en bueno el $10,3 \%, 14,2 \%$ y $29,5 \%$; en el parámetro regular $0,8 \%, 4,7 \%$ y $7,9 \%$; habiendo un mínimo porcentaje como malo para el Bolívar del $0,8 \%$ y Hualtaco el $4,7 \%$. 
Figura $N^{\circ}$ 11. Criterio Imagen: Emplatado.

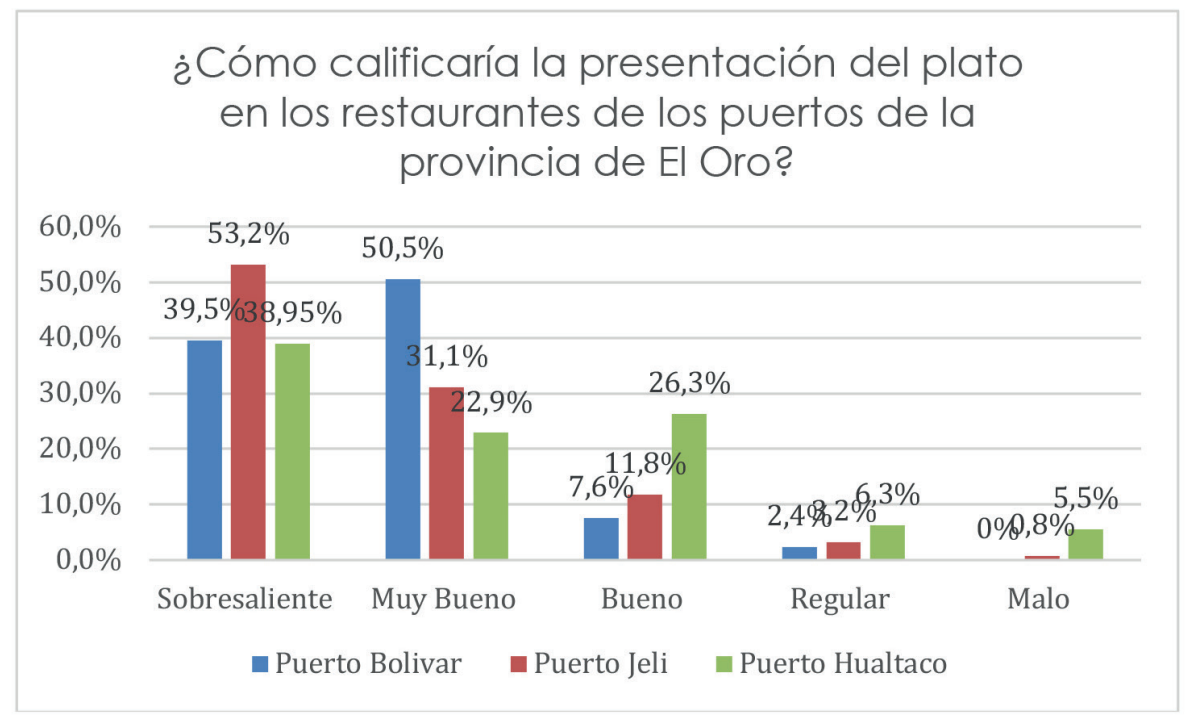

Fuente: Elaborado por autores. Trabajo de campo 2019.

En la actualidad, con la creciente competencia, se hace más evidente la frase "la comida entra por los ojos", es por ello que grandes compañías multimillonarias como: McDonald y KFC, entre otras, invierten en la imagen de su producto, muchas de las veces en restauración la manera en la que se presenta la comida puede ser decisivo a la hora de atraer clientes. Pérez (2012), menciona tres factores que se deben tomar en cuenta para el emplatado del marisco: naturaleza y tipo de espécimen, su proceso de preparación y el tipo de servicio empleado, en base a ello la presentación y decoración puede ser variada, ya sea por gustos del chef o políticas del establecimiento, el modelo y color del plato, - las características de la salsa o guarnición. Habiendo un calificativo de sobresaliente para Puerto Bolívar del 39,5\%, Puerto Jeli del 53,2\% y Puerto Hualtaco del 38,95\%; como muy bueno tienen el $50,5 \%$, 31,1\% y $22,9 \%$; en bueno poseen el $7,6 \%, 11,8 \%$ y $26,3 \%$; en cambio como regular porcentajes de $2,4 \%, 3,2 \%$ y $6,3 \%$; dejando resultados del $0 \%$, $0,8 \%$ y $5,5 \%$ en malo.

Además, en el criterio de valorización de Precio muestra lo siguiente:

Figura No 12. Criterio Precio: Relación plato-precio.

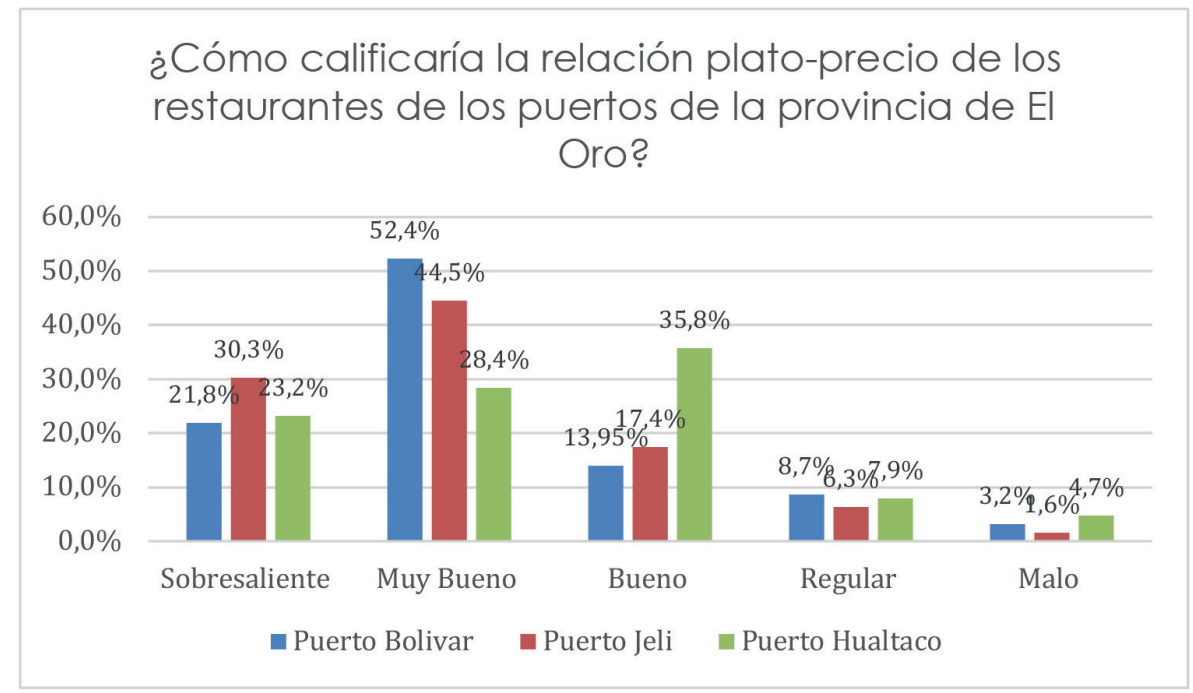

Fuente: Elaborado por autores. Trabajo de campo 2019. 
Según Gallego (1998), el precio promedio de la gastronomía en un sitio de comida debe estar acorde a la categoría del establecimiento. Sin embargo, cierto número de restaurantes en los puertos de la provincia de El Oro no se encuentran debidamente registrados en el catastro turístico y no poseen una categoría específica, a pesar de ello la relación plato-precio según los datos obtenidos en la presente investigación indican que en Puerto Bolívar el 21,8\% lo considera sobresaliente, asimismo en Puerto Jeli el $30,3 \%$ y en Puerto Hualtaco un 23,2\%; como muy bueno tienen el $52,4 \%, 44,5 \%$ y $28,4 \%$; en cambio en bueno $13,95 \%, 17,4 \%$ y $35,8 \%$; en regular poseen $8,7 \%, 6,3 \%$ y $7,9 \%$; para obtener como malo $3,2 \%, 1,6 \%$ y $4,7 \%$.

\section{CONCLUSIONES}

A pesar de obtener resultados positivos en el criterio Servicio, los establecimientos de comida, deberían enfocarse más en sus empleados, a través de capacitaciones o incentivos que estimulen mejorar la atención al cliente, la cual debe ser ágil desde el ingreso hasta la salida del comensal, ello también incluye el tiempo de espera determinado para la preparación de los aperitivos, el cual debe mantenerse entre 15 a 20 minutos. Asimismo, al momento de la facturación o cancelación del consumo es recomendable que no haya solo la modalidad de pago de efectivo, sino también otras formas de pago, tales como: tarjeta de crédito y transferencia, entre otros; como también que el tiempo estimado de facturación debe ser de 5 minutos, caso contrario el establecimiento estaría mostrando una mala distribución de tareas asignadas para cada colaborador.

Se pudo determinar que el criterio Producto tiene índices altos de satisfacción, principalmente porque la producción de su gastronomía es en base a mariscos frescos, debido a la aproximación que tienen con el manglar, sin embargo, sería propicio que adopten nuevas formas de preparación y cocción enfocados a un tipo de cocina internacional, para de esta manera acaparar nuevos nichos de mercado sin perder su enfoque tradicional, que es su sabor, textura y aroma el cual varía entre los tres puertos, pero con una notable diferencia Jeli presenta el $0 \%$ de insatisfacción.

Puerto Hualtaco en el criterio instalación y mobiliaria, con respecto a los resultados, adquirió el calificativo más bajo, por ende, debería enfocarse en mostrar una mejor pulcritud para sus comensales y aprovechar su entorno que está enlazado a elementos naturales, de igual manera, los comensales no se sienten totalmente cómodos con el espacio que tienen los restaurantes, por ello se recomienda de forma opcional una restructuración de las mesas, mobiliarios o accesorios decorativos que posea, y organizarlos acorde al área que tenga el sitio.

Con respecto al criterio Seguridad, se concluyó que debe haber un mayor control policial e interés por parte de los restaurantes en generar confianza al cliente, a través de medidas de prevención ante la delincuencia, además, que es necesario establecer sitios de estacionamiento concretos y seguros, debido a que esto crea parqueaderos informales.

En el criterio Imagen se identificó que la identidad corporativa de Puerto Hualtaco es débil frente a la competencia, ello provoca que la primera impresión que tenga el comensal acerca del lugar no siempre sea positiva, por esta razón es recomendable establecer estándares de conducta, vestuario y ambiente laboral, para que los colaboradores las recepten y difundan.

Con relación al criterio Precio, se puede corroborar que el valor estándar de un plato a base de mariscos es de 8 dólares, esto incrementa acorde al tipo de espécimen que se desee, los tres puertos poseen porcentajes similares, por ende, los encuestados establecen que la relación entre lo que pagan y lo que obtienen es la adecuada. 


\section{REFERENCIAS}

Aguilar , J., Yepez , V., Esteban , V., \& Serra , J. (1999). Calidad y gestión de recursos costeros. Jornadas Españolas de Ingeniería de Costas y Puertos, 877-890.

Besanilla , T., Rodríguez , H., \& Ponce , M. (2012). Factores que influyen en el comportamiento del consumidor. EUMED. Obtenido de http://www.eumed. net/ce/2012/dhi.pdf

Bull, A. (2003). Congestion de transito. Santiago: CEPAL. Obtenido de https://repositorio.cepal.org/ bitstream/handle/11362/27813/6/S0301049 es.pdf

Crespo, J. (09 de 02 de 2019). Militares refuerzan seguridad en El Oro ante incremento de asaltos y muertes . Expreso . Obtenido de https://www.expreso. ec/actualidad/militares-seguridad-fuerzasarmadaspolicianacional-eloro-LF2623850

Delgado , R. (2001). Comida y cultura: identidad y significado en el mundo Contemporáneo. Estudios de Asia y África, 82-108. Obtenido de https://www. redalyc.org/pdf/586/58636104.pdf

Estrada, W. (2007). Servicio y atencion al cliente. Perú. Obtenido de http://historico.pj.gob.pe/CorteSuprema/cij/documentos/ServicioAtencionCliente110708.pdf

Galán , J. (2008). Gestión Vectorial de la Imagen Corporativa. Redalyc(65). Obtenido de https://www.redalyc. org/articulo.oa?id=199520724022

Gallego, J. (1998). Manual práctico de restaurante. Madrid :Paraninfo.
INEC. (2010). ecuadorencifras. Recuperado el 11 de 09 de 2018, de http://www.ecuadorencifras.gob.ec/ informacion-censal-cantonal/?fbclid=IwAR2QCaRxIDHyc5qPfLm_ZuuxDykfAMUb5aM9E3-MwluQ2g3E4ZqJFB72zsE

Maldonado , L. (2015). Los medios de pago, un paisaje en movimiento. Madrid: Centro del Sector Financiero de PwC e IE Business School. Obtenido de https://www.pwc.es/es/publicaciones/financiero-seguros/assets/medios-pago-paisaje-movimiento.pdf

Narváez, M., \& Fernández, G. (2010). El turismo desde la perspectiva de la demanda. SCIELO, 175-183. Obtenido de http://www.scielo.org.co/pdf/rudca/ v13n2/v13n2a20.pdf

Oliveira , B. (2010). Determinantes de la satisfaccion del turista. Scielo, 229-242. Obtenido de http://www. redalyc.org/pdf/1807/180717677013.pdf

Pérez , V. (2012). Elaboraciones básicas y platos elementales con pescados, crustáceos y moluscos. España: Paraninfo.

Roberts, T., \& Stadler, K. (2009). Pescados y Mariscos en Virginia. VirginiaTech, 9. Obtenido de https:// www.pubs.ext.vt.edu/content/dam/pubs_ext_vt_ edu/348S/348-961S/348-961S_pdf.pdf

Rubio , R. (2014). Aplicación de normas y condiciones higiénico-sanitarias en restauración. Vigo: Ideaspropias. Obtenido de https://issuu.com/ideaspropiaseditorial/docs/978-84-9839-501-3_caa52855b1adb7 